\title{
Yellow-red and nonobtuse refinements of planar triangulations
}

Sergey Korotov and Jacek Stańdo 


\title{
YELLOW-RED AND NONOBTUSE REFINEMENTS OF PLANAR TRIANGULATIONS
}

\author{
Sergey Korotov \\ Department of Mathematical Information Technology \\ University of Jyväskylä, FIN-40351 Jyväskylä, Finland \\ korotov@mit.jyu.fi \\ JACEK STAŃDO \\ Institute of Mathematics, Technical University of Łódź \\ Al. Politechniki 11, 90-924 Łódź, Poland \\ standoj@ck-sg.p.lodz.pl
}

[Received: January 15, 2002]

\begin{abstract}
A new yellow-red refinement of nonobtuse triangles, combining standard red and yellow refinements, is proposed. This approach is also adapted for the construction of nonobtuse global refinement of an initial triangulation in the case when some obtuse triangles in it are created by a grid generator.
\end{abstract}

Mathematical Subject Classification: 65N30, 65N50

Keywords: nonobtuse triangulation, polygonal domain, finite element method, discrete maximum principle, maximum angle condition, red and yellow refinements, grid generation

\section{Introduction}

In this paper, we consider conforming (planar) triangulations of a polygon into triangles, i.e., the union of all triangles of any particular triangulation is always equal to our polygon, and any two different triangles in any (particular) triangulation may only have a common edge, or a common vertex, or no common points (cf. [11]). In most cases namely such conforming triangulations are used in finite element modelling and finite element analysis.

A planar triangulation is called nonobtuse if all interior angles in all triangles of the triangulation are less than or equal to $\frac{\pi}{2}$. This condition was first introduced in [4] and [15], where it was particularly used to prove the discrete maximum principle for the piecewise linear finite element approximations to the Poisson equation with the Dirichlet boundary condition. Later this condition was imposed on the interior angles between faces of tetrahedra in tetrahedral partitions, and again used to prove the discrete maximum principle for the finite element solutions to a class of nonlinear elliptic problems for which the continuous maximum principle holds, see [12]. Obviously, a similar question, under which conditions on the triangulations used the 


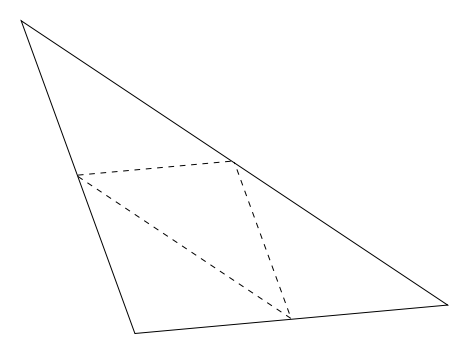

a) red refinement

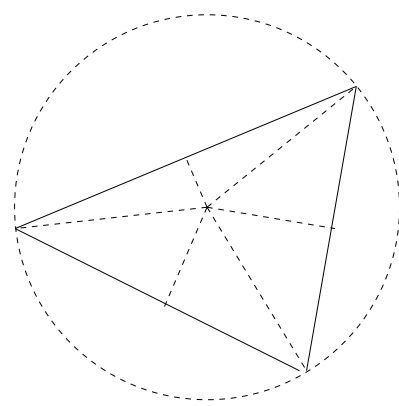

b) yellow refinement

Figure 1

discrete maximum principle holds, naturally appears in the case of parabolic problems. For these problems there are also some other important qualitative properties closely related to the preservation of the maximum principle, we refer to [5], [6], and [14] in this respect.

Note that in nonobtuse triangulations, all triangles satisfy the maximum angle condition with the same constant, $\frac{\pi}{2}$. This condition is very important for the finite element analysis, since the optimum interpolation properties of the finite elements are preserved [11, Chapt. 4].

The construction of a conforming triangulation of a polygon into (arbitrary) triangles is, obviously, simple; moreover, there are proofs of the existence of nonobtuse triangulations of polygons [1], [7]. Thus, having a nonobtuse initial triangulation, and then applying the red refinement [2] and/or the yellow refinement [9] (see Figure 1), we can easily build a family of globally refined triangulations where no obtuse angles occur.

Nevertheless, in practice, the initial (coarse) triangulation of the polygonal domain is performed by a grid generator, and it is most probable that this initial triangulation is not nonobtuse. In this paper, we propose, first, a new way of refinement of a single nonobtuse triangle, combining the standard yellow and red refinement techniques (which we will call the yellow-red refinement). Thus, a nonobtuse triangulation can, in fact, be globally refined using red and/or yellow and/or yellow-red refinements for its triangles (in any desired combination). Further, evolving the approach used for yellow-red refinement, we also analyse a possibility of refining globally the given triangulation, initially containing several isolated obtuse triangles (cf. Figure 2), into the conforming nonobtuse triangulation. In particular, we present the algebraic conditions on the coordinates of vertices of a quadrilateral formed by obtuse and nonobtuse triangles in the triangulation (where those two triangles are adjacent along the longest side of the obtuse triangle), under which it is possible to refine the quadrilateral into nonobtuse subtriangles (cf. Figure 4a). In the partition proposed in this paper, the midpoints of non-common sides of the initial triangles and the center of the circumscribed circle around the obtuse triangle, which is inside of the quadrilateral, are used. 
Thus, if the conditions presented are satisfied for all the obtuse triangles and nonobtuse triangles adjacent to them (in the above-described manner), occurring in the initial triangulation, we can conformly (globally) refine the given initial triangulation so that the next (refined) triangulation (after one refinement step) already becomes nonobtuse.
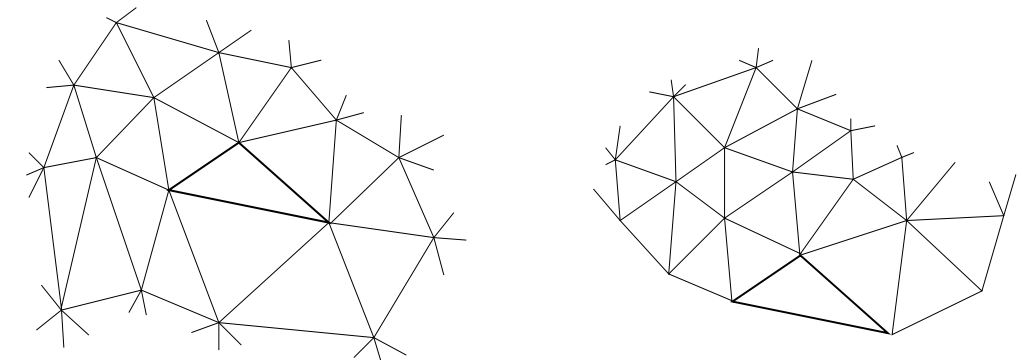

a) obtuse triangle is inside of solution domain b) obtuse triangle is adjacent to the boundary

Figure 2

It is also worth mentioning here, that the nonobtusity condition is only sufficient to prove the discrete maximum principle: it is possible to observe the validity of this principle also in situations when some of the interior angles in the triangulation are slightly obtuse. This issue, leading to so-called weakened acute type conditions, is addressed in [13] and [10] in the two- and three-dimensional cases, respectively.

Other known refinements of triangles are green [2], and blue [8], they are used for local refinement procedures. For a comprehensive survey of the results on the grid generation issues, we refer to monograph [3].

\section{Nonobtuse triangle and yellow-red refinement}

2.1. Criterion for a nonobtuse triangle. In this section, we give a simple criterion for a triangle to be nonobtuse, and introduce the idea of yellow-red refinement.

We present a simple criterion, which employs the coordinates of vertices of a triangle, to decide whether this triangle is nonobtuse. From now on, $A B C$ always stands for a triangle with vertices $A=\left(x_{A}, y_{A}\right), B=\left(x_{B}, y_{B}\right)$, and $C=\left(x_{C}, y_{C}\right)$ (see Figure 3).

Lemma 1. Triangle $A B C$ is nonobtuse if and only if the following three inequalities hold

$$
\begin{aligned}
& \left(x_{A}-2 x_{B}+x_{C}\right)^{2}+\left(y_{A}-2 y_{B}+y_{C}\right)^{2} \geq\left(x_{C}-x_{A}\right)^{2}+\left(y_{C}-y_{A}\right)^{2}, \\
& \left(x_{C}-2 x_{A}+x_{B}\right)^{2}+\left(y_{C}-2 y_{A}+y_{B}\right)^{2} \geq\left(x_{B}-x_{C}\right)^{2}+\left(y_{B}-y_{C}\right)^{2}, \\
& \left(x_{B}-2 x_{C}+x_{A}\right)^{2}+\left(y_{B}-2 y_{C}+y_{A}\right)^{2} \geq\left(x_{A}-x_{B}\right)^{2}+\left(y_{A}-y_{B}\right)^{2} .
\end{aligned}
$$

Proof. Conditions (2.1a)-(2.1c) imply that $|B K| \geq \frac{1}{2}|A C|,|A L| \geq \frac{1}{2}|C B|,|C M| \geq$ $\frac{1}{2}|A B|$, where $K, L, M$ are midpoints of $A C, C B, A B$, respectively. Geometrically, 


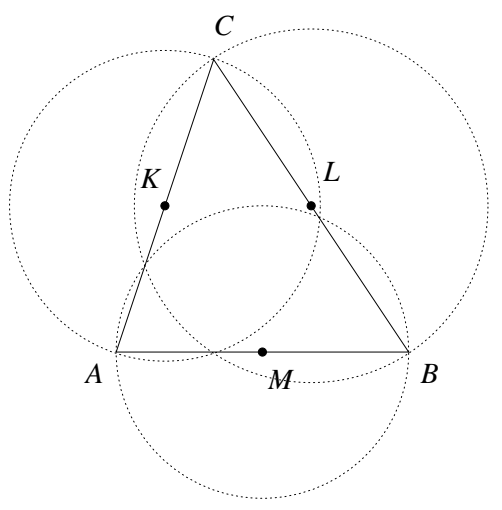

a) acute triangle $A B C$

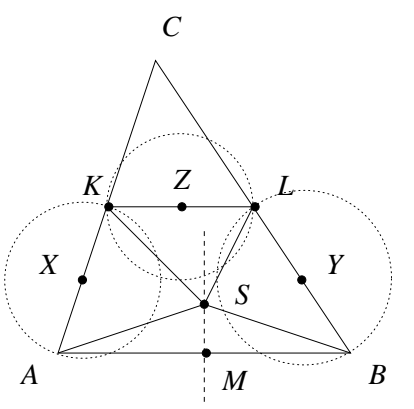

b) yellow-red refinement

Figure 3

it means that (2.1a) is equivalent to the case when vertex $B$ does not lie inside of a circle having $A C$ as its diameter (and $K$ as its center), i.e., the angle at $B$ is nonobtuse; the same holds for vertices $A$ and $C$, from (2.1b) and (2.1c), respectively (cf. Figure 3a).

2.2. Yellow-red refinement. The idea of red refinement from [2] (cf. Figure 1a) consists of the usage of the midpoints of sides of the triangle: connecting them, we get four subtriangles similar to the original one; red refinement can be applied to any triangle.

The idea of yellow refinement was recently introduced in [9] for tetrahedra and their faces. It essentially uses the assumption that the center of the circumscribed circle of the given triangle lies in the closure of the triangle. Using this center and the midpoints of sides of the triangle, we get partition into four or six right subtriangles, depending on the value of the maximum angle in the given triangle (cf. Figure 1b). Thus, yellow refinement can only be applied to a nonobtuse triangle.

Now, we show that it is also possible to combine both techniques inside of a nonobtuse triangle. The idea of such a construction is explained (in terms of Figure $3 \mathrm{~b}$ ) as follows: let $K, L, M$ be defined as before, the dashed line via point $M$ be the mid-perpendicular to side $A B$, points $X, Y, Z$ be the midpoints of $A K, B L, K L$, respectively (these three points are also centers of three dotted circles having $A K$, $B L$, and $K L$ as their diameters). If we can take point $S$ from trapezoid $A K L B$ $(\subset A B C)$, lying on the mid-perpendicular via $M$, so that it does not lie inside of all three (dotted) circles, then triangle $A B C$ is, obviously, decomposed into six nonob-

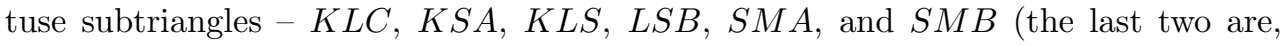
moreover, right ones at the vertex $M)$.

Remark 1. In general, there exist many choices for the position of point S. Nevertheless, there may occur a situation when the only possible choice of $S$ is to be at 
point $M$, and it only happens if $A B C$ is the right triangle. In this case, the yellow-red refinement coincides with the standard red refinement of triangle $A B C$.

2.3. Nonobtuse refinements. In this section, we discuss the issue of the construction of (refined) nonobtuse triangulations from the initially generated one, containing obtuse triangles; it is, particularly, shown how the yellow-red refinement technique can be adapted for this purpose.

2.4. Nonobtuse refinement of two adjacent triangles. The approach, used in Section 2.2, can be adapted to the problem of partitioning the triangulations with obtuse triangles. For this purpose, we make the following natural observation - we need not always make the refinement only in a single triangle, we can also perform the refinement, for example, for a pair of adjacent triangles.

To show how this observation and the yellow-red refinement can be employed, we assume (in denotations of Figure 4a) that triangle $A D B$ is obtuse at $D=\left(x_{D}, y_{D}\right)$ and triangle $A B C$ is nonobtuse as before. Also, let $X=\left(x_{X}, y_{X}\right), Y=\left(x_{Y}, y_{Y}\right)$, and $Z=\left(x_{Z}, y_{Z}\right)$ be midpoints of $A K, B L$, and $K L$, respectively.

Lemma 2. The coordinates of $X, Y$, and $Z$ are given by the formulae

$$
\begin{aligned}
x_{X} & =\frac{3}{4} x_{A}+\frac{1}{4} x_{C}, & y_{X} & =\frac{3}{4} y_{A}+\frac{1}{4} y_{C} ; \\
x_{Y} & =\frac{3}{4} x_{B}+\frac{1}{4} x_{C}, & y_{Y} & =\frac{3}{4} y_{B}+\frac{1}{4} y_{C} ; \\
x_{Z}=\frac{1}{2} x_{C} & +\frac{1}{4} x_{A}+\frac{1}{4} x_{B}, & y_{Z} & =\frac{1}{2} y_{C}+\frac{1}{4} y_{A}+\frac{1}{4} y_{B} .
\end{aligned}
$$

Further, let $S=\left(x_{S}, y_{S}\right)$ be the center of the circumscribed circle of $A D B$, it stands at the intersection of three mid-perpendiculars to sides $A D, D B$ and $A B$, and lies outside of $A D B$, since it is obtuse.

Lemma 3. The coordinates of $S$ are given by the formulae

$$
\begin{aligned}
& x_{S}=\frac{\left(y_{B}-y_{D}\right)\left(x_{A}^{2}+y_{A}^{2}-x_{D}^{2}-y_{D}^{2}\right)+\left(y_{D}-y_{A}\right)\left(x_{B}^{2}+y_{B}^{2}-x_{D}^{2}-y_{D}^{2}\right)}{2 \cdot\left[\left(x_{A}-x_{D}\right)\left(y_{B}-y_{D}\right)-\left(x_{B}-x_{D}\right)\left(y_{A}-y_{D}\right)\right]}, \\
& y_{S}=\frac{\left(x_{D}-x_{B}\right)\left(x_{A}^{2}+y_{A}^{2}-x_{D}^{2}-y_{D}^{2}\right)+\left(x_{A}-x_{D}\right)\left(x_{B}^{2}+y_{B}^{2}-x_{D}^{2}-y_{D}^{2}\right)}{2 \cdot\left[\left(x_{A}-x_{D}\right)\left(y_{B}-y_{D}\right)-\left(x_{B}-x_{D}\right)\left(y_{A}-y_{D}\right)\right]} .
\end{aligned}
$$

The calculation in both lemmas is straightforward, therefore omitted.

Further, the following theorem holds, where $|\cdot|$ denotes the standard distance between two points in plane.

Theorem 1. Let a triangle $A B C$, adjacent to an obtuse triangle $A D B$, be nonobtuse. If

$$
\begin{aligned}
{\left[\left(x_{C}-\right.\right.} & \left.\left.x_{A}\right)\left(y_{A}-y_{B}\right)+\left(y_{C}-y_{A}\right)\left(x_{B}-x_{A}\right)\right] \times \\
& \times\left[\left(x_{S}-\frac{1}{2}\left(x_{C}+x_{A}\right)\right)\left(y_{A}-y_{B}\right)+\left(y_{S}-\frac{1}{2}\left(y_{C}+y_{A}\right)\right)\left(x_{B}-x_{A}\right)\right] \leq 0
\end{aligned}
$$




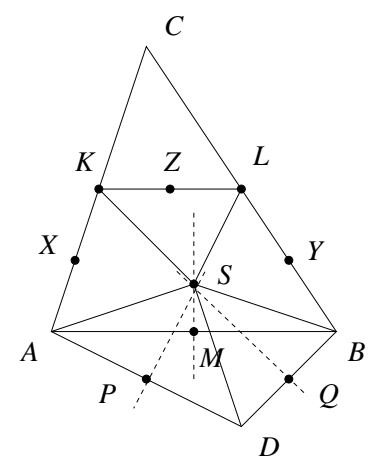

a) refinement of quadrilateral is possible

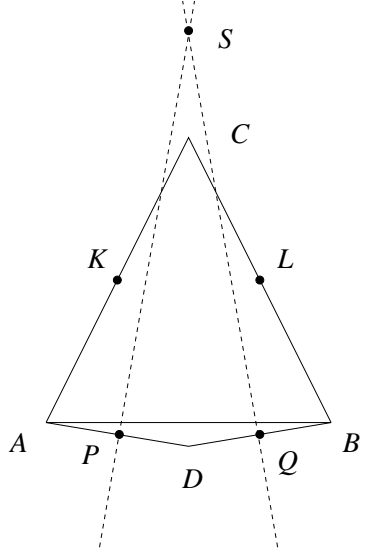

b) refinement of quadrilateral is not possible

Figure 4.

and

$$
|S X| \geq \frac{1}{4}|A C|, \quad|S Z| \geq \frac{1}{4}|A B|, \quad|S Y| \geq \frac{1}{4}|B C|,
$$

where coordinates of points $S, X, Y$, and $Z$ are given by (3.1)-(3.2), then subtriangles $K L C, K S A, K L S, L S B, S A P, S P D, S D Q$, and $S Q B$ are nonobtuse (the last four are, moreover, right triangles). Above, $P$ and $Q$ are the midpoints of sides $A D$ and $D B$, respectively (see Figure 4a).

Proof. Condition (3.3) means that points $C$ and $S$ are in different half-planes with respect to straight-line $K L$, i.e., $S$ lies in the trapezoid $A K L B$. Further, condition (3.4) means that point $S$ is outside of three circles defined as in Figure $3 \mathrm{~b}$. Thus, all the above mentioned subtriangles make a nonobtuse partition of a quadrilateral $A D B C$.

Remark 2. Obviously, the conditions of Theorem 5 cannot be fulfilled in a general case. For example, in Figure 4b, two adjacent (obtuse and nonobtuse) triangles are such that the point $S$ is completely outside of the quadrilateral made by the triangles. Therefore, a partition of the quadrilateral in the manner, proposed in Theorem 5, is not possible.

Remark 3. One of the other possibilities to get nonobtuse triangles, particularly, for the case of Figure $4 \mathrm{~b}$, would be to use a swapping of diagonals in the quadrilateral. Thus, in the decomposition of $A D B C$ we can employ two triangles $A C D$ and $B C D$ (which could be both nonobtuse) instead of $A B C$ and $A B D$. However, the analysis of such an approach, is beyond the scope of the present paper. 
2.5. On nonobtuse refinements of coarse triangulations. In this subsection, we shortly discuss how the ideas from the previous subsection can be used to make the nonobtuse triangulation from the coarse triangulation, with obtuse triangles in it, built by the grid generator.

So far, various refinement techniques proposed - red, yellow, and yellow-red are only applied to the single triangle. As we mentioned above, the red refinement, applied to the obtuse triangle, gives obtuse subtriangles; the other two techniques can only be applied to nonobtuse triangles by their definitions. However, the yellow-red technique can be used for the union of adjacent triangles, one of which is obtuse and the other is nonobtuse, as it was shown in Section 3.1.

Thus, we can make a refinement of the initial (generated) triangulation in the following manner. Using the criterion of Section 2.1, we check whether a triangle taken from the mesh is nonobtuse; and, thus, mark all obtuse triangles. If the (marked) obtuse triangle is adjacent to the boundary along its longest edge (see Figure 2b), we let the perpendicular from the interior vertex of this triangle onto the boundary, and add a new node and two right-angled triangles to the triangulation.

Further, if we have an obtuse triangle inside of the solution domain (see Figure 2a), we consider the quadrilateral formed by it and adjacent to it and try to apply the technique of Section 3.1.

Remark 4. A similar question - how to build nonobtuse tetrahedral triangulations from triangulations containing some tetrahedra with obtuse interior angles between faces, naturally appears in the three-dimensional case. To the authors' knowledge this is an open problem. Another related open question is how to make local nonobtuse refinements of planar and tetrahedral triangulations. It is also interesting to make research on which geometrical properties of triangulations in the two- and threedimensional cases provide the validity of the discrete maximum principle for parabolic problems.

\section{Numerical experiments}

Numerical experiments on the effectivity of the proposed technique have been performed using Matlab in Intel $700 \mathrm{MgHz} / 128 \mathrm{RAM}$. We (pseudo)randomly generated 100000 times the coordinates of vertices $A, B, C, D$ (the time needed for this was 1131 seconds): the situation when one of triangles $A B C$ or $A B D$ had an obtuse angle at the vertex $C$ or $D$, and the other triangle had a nonobtuse angle at $D$ or $C$, respectively, occured 17235 times. For those 17235 "undesirable" cases, we could successfully apply 2353 times the result of Theorem 5 (the time needed for that was 56 seconds). Also, the swapping procedure of Remark 7 was "successful" for 2488 cases from those 17235 "undesirable" cases (time costs for this checking was 24 seconds), and there was no intersection between "successful" cases of Theorem 5 and Remark 7 (the time needed for this was 22 seconds).

Another interesting result was that the situation, when both angles $C$ and $D$ in the triangles $A B C$ and $A B D$ were obtuse, occurred 11563 times, and we could 
"successfully" apply the swapping procedure from Remark 7 to 2856 cases from those 11563 (time costs in this experiment was 119 seconds).

Acknowledgement. The authors are grateful to Prof. Michal Křížek for valuable comments on the paper. The first author was supported by Grant no. 49051 of the Academy of Finland, the second author was supported by the Academy of Poland.

\section{REFERENCES}

[1] Baker, B. S., Grosse, E. and RAfFerty, C. S.: Nonobtuse triangulation of polygons, Discrete \& Computational Geometry, 3, (1988), 147-168.

[2] Bank, R. E.: PLTMG: A Software Package for Solving Partial Differential Equations: Users' Guide 7.0, SIAM, Philadelphia, 1994.

[3] Carey, G. F.: Computational Grids. Generation, Adaptation, and Solution Strategies, Series in Computational and Physical Processes in Mechanics and Thermal Sciences. Taylor \& Francis, Washington, DC, 1997.

[4] Ciarlet, P. G. and Raviart, P. A.: Maximum principle and uniform convergence for the finite element method, Computer Methods in Applied Mechanics and Engineering, 2, (1973), 17-31.

[5] FARAGó, I.: Regular and exponential convergence of difference schemes for the heatconduction equation, Computers \& Mathematics with Applications, 38, (1999), 71-77.

[6] FARAgó, I. and TARvainen. P.: Qualitative analysis of one-step algebraic models with tridiagonal Toeplitz matrices, Periodica Mathematica Hungarica, 35 (3), (1997), 177 192.

[7] Gerver, J. L.: The Dissection of a Polygon into Nearly Equilateral Triangles, Geometriae Dedicata, 16, (1984), 93-106.

[8] Kornhuber, R. and Roitzsch, R.: On adaptive grid refinement in the presence of internal or boundary layers, Impact of Computing in Science and Engineering, 2, (1990), 40-72.

[9] Korotov, S. and Kř́̌žEK, M.: Acute Type refinements of tetrahedral partitions of polyhedral domains, SIAM Journal on Numerical Analysis, 39, (2001), 724-733.

[10] Korotov, S., Kř́̇̌žer, M. and NeittaAnmäki, P.: Weakened Acute type condition and the discrete maximum principle, Mathematics of Computation, 70, (2000), 107-119.

[11] KřÍžEK, M. and NeitTAanmÄKI, P.: Mathematical and Numerical Modelling in Electrical Engineering: Theory and Applications, Kluwer Academic Publishers, Dordrecht, 1996.

[12] KřÍžEK, M. and QUN LiN: On diagonal dominance of stiffness matrices in 3D, EastWest Journal on Numerical Mathematics, 3(1), (1995), 59-69.

[13] Ruas Santos, V.: On the strong maximum principle for some piecewise linear finite element approximate problems of non-positive type, Journal of the Faculty of Sciences. University of Tokyo. Section IA. Mathematics, 29, (1982), 473-491.

[14] Stoyan, G.: On a maximum principle for matrices, and on conservation of monotonicity, Zeitschrift für Angewandte Mathematik und Mechanik, 2, (1982), 375-381.

[15] Strang, G. and Fix, G. J.: An Analysis of the Finite Element Method, Prentice Hall, Englewood Cliffs, N. J., 1973. 